\title{
Involuntary psychiatric admission - a comparison between legal frameworks
}

\author{
Mirona Letitia Dobri, Alina-Ioana Voinea, \\ Ionuţ-Dragoş Rădulescu, Petronela Nechita
}

\begin{abstract}
Mirona-Letiţia Dobri - M.D., Resident in Psychiatry,"Socola" Institute of Psychiatry, Iaşi, România

Alina-Ioana Voinea - M.D., Resident in Psychiatry,"Socola" Institute of Psychiatry, Iaşi, România Ionuţ-Dragoş Rădulescu - M.D., Resident in Psychiatry,"Socola" Institute of Psychiatry, Iaşi, România

Petronela Nechita - M.D., Ph. D., Senior Psychiatrist,"Socola” Institute of Psychiatry, Iaşi, România
\end{abstract}

\begin{abstract}
Persons suffering from psychiatric disorders are included in a category of patients considered as vulnerable. Therefore, mental health practitioners walk a fine line around various ethical aspects related to these patients' care. Consent is a fundamental part of every medical act, which makes admission and treatment without consent a delicate issue surrounded by much debate. Despite efforts to unify the approach to involuntary admission of the psychiatric patient, the mental health acts vary greatly between different countries. This review describes legal frameworks applicable in countries across Europe and America, underlining the legal procedures and the most often used criteria that qualify the patient for involuntary admission. These criteria include the presence of a psychiatric disorder, the dangerousness criterion and the need for treatment. The differences in concept regarding the necessary clinical criteria for a mental disorder to be included on the list of diagnoses subjectable to involuntary admissions create an uneven ethical and moral picture regarding the status of the psychiatric patient amongst the different legislation frameworks, thus predisposing him to other possible issues, such as discrimination. An important aspect in the procedure of involuntary admission of the patient with psychiatric disorders consists of keeping a balance between the right to self-determination and the care duties of the medical personnel. Therefore, managing psychiatric patients involuntarily admitted proves a challenge for mental health practitioners and society, as a whole, as it touches multiple ethical and social issues.
\end{abstract}

\section{KEY WORDS:}

Mental disorders, involuntary admission, informed consent, confidentiality 


\section{INTRODUCTION}

Mental health has been a much-discussed subject through the years all around the world and constitutes a concept that has been consistently redefined through the passing of time and which varies greatly in both definition and management depending on the culture and history of the place involved. Globally, a fluctuance can be observed regarding the medical, social and legal approach to each disorder labelled by the term "mental health issue", strategy which contributes to the yielding of vastly different statistics even between countries of similar culture and socio-economic status. Overall, World Health Organization cites that grossly a fourth of the world's population suffers or will suffer from a mental health issue in the future. $(1,2)$ These numbers bring attention to a very important element that comes forward in the psychiatric care process, the principle of autonomy.

Medical ethics discusses four fundamental principles for patient care: the principle of respect for the patient's autonomy, the principle of nonmaleficence, beneficence and justice. Together with many other rules and laws designed to regulate patient care, these are the basis of the doctor -patient relationship and if respected they can bring a better prospect for the resolution of the patient's problem. However, in psychiatry, there is a fine line between respecting and breaking these principles, which creates a challenge from the point of view of medical care as well as the legal framework. In the service of preserving the four principles above mentioned, there is the notion of informed consent, a mandatory requirement for a good psychiatric medical assistance.

Consent is a specific, legal act, necessary to be expressed in writing, as a result of a complete patient briefing. The patient should receive information on the need for hospitalization, treatment, diagnosis, benefits and risks. The doctor must use a language appropriate to the patient's level of training. $(1,2)$

In psychiatric care there are two ways of hospitalization: one with consent (voluntary) and involuntary admission. In psychiatric clinics, involuntary admission of patients with psychiatric disorders applies only after all attempts at voluntary admission have been exhausted. (3) Involuntary admission is one of the most controversial issues in the field of mental health. Our aim is to document how the involuntary admission process varies in different medical systems and how it impacts the distinct aspects of psychiatric care.

\section{ASPECTS OF INVOLUNTARY ADMISSION OF THE PSYCHIATRIC PATIENT}

Involuntary psychiatric hospitalization constitutes a factor that influences in a great way the maintenance of the four principles. An important aspect often discussed in cases of involuntary admission is the maintenance of a balance between the right to selfdetermination and care responsibilities. The principle of autonomy is sometimes breached in favor of the principle of beneficence, when is considered that involuntary admission is a necessary measure to protect the patients or society, for instance when mental disorder patients pose a risk of harm to themselves or to those around them. $(4,5)$

Thus, time has constructed the notion of "dangerousness criteria" for mandatory admission, which constitute elements that indicate that an individual with a psychiatric disorder poses a risk to himself or society that can be eliminated by hospital admission. These criteria pose a series of ethical dilemmas, including that of the real possibility of predicting dangers. Respect for autonomy has become a priority in recent years, by getting the patient more and more 


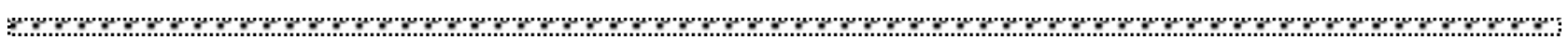
involved in making decisions about his or her health. $(4,6)$

The subject of involuntary admission is a controversy, because coercive measures on psychiatric patients are traumatizing and stigmatizing experiences, but at the same time, patient access to treatment, even if it is mandatory has multiple future benefits concerning the improvement of quality of life and social functioning. (1) A classic example of this is in patients with schizophrenia. As many of these do not have access to psychiatric treatment or are not compliant to medication, it is debated whether mandatory treatment improves quality of life and social functioning on the long term, as opposed to stigmatization. (6) Multiple hospitalizations lead to isolation, and in time to institutionalization and labeling, thus respecting a psychiatric treatment will reduce repeated hospitalization, aggression (8) and antisocial behaviors. The statistic shows that stigmatization of psychiatric patient decreases with the diminishing of symptoms and improvement of social functioning.

Among the negative effects of stigmatization are reduced addressability to receive treatment, rejection by friends and family, discrimination in society, difficulty in finding employment and housing, verbal and physical violence, etc. $(9,10)$, increased risk of suicide. Social integration of the psychiatric patient though society's awareness of the devastating effects of stigmatization would be a great way of reducing the above-mentioned issues but is a difficult target to achieve because of its grandeur of scope, and a great number of government-funded and community-upheld organizations and institutions would need to work in synergy to reaching this goal.

COMPARATIVE PERSPECTIVES OF INVOLUNTARY PSYCHIATRIC ADMISSIONS WITHIN DIFFERENT MENTAL HEALTH ACTS

All the numerous and complex aspects of involuntary hospitalization and treatment and the inherent ripple effects on the psychiatric patient's status within the society create a potential pattern of vulnerability that throughout the years has been outlined on various occasions. Therefore, this issue has seen the necessity to construct several means of protection for this type of patient from both the medical and legal perspective. Tremendous effort has been made by World Health Organization to provide a unique guideline of care and procedure in these patients. There are many international guidelines available to provide context, of which we mention the Principles for the Protection of Persons with Mental Illness (or MI Principles, 1991), the European Convention for the Protection of Human Rights and Fundamental Freedoms (1950), The Declaration of Hawaii (1983), and the Ten Basic Principles for Mental Health Law published by the World Health Organization (WHO). (7, 11) Presently the Mental Health Acts and Laws implemented vary by country due to multiple factors such as cultural traditions and restrictions, economies and human resources. (7) Differentiated criteria are applied to qualify a patient for involuntary psychiatric admissions, leading to a lack of uniformity in statistics.

To define the criteria for involuntary psychiatric admission is a very delicate process, attempted by WHO with the checklist for Involuntary Admission and Treatment. (7) It specifies that the patient must be suffering from a severe mental disorder, mandatory treatment is necessary in the service of the patient's health and safety or in the protection of other persons. 
However, these criteria are not adopted by all healthcare systems.

\section{Presence of a Psychiatric Disorder}

All mental health care acts specify that the patient must be suffering from a mental disorder, but the definition is variable. Some countries use the restrictive term of "severe mental disorder", others use a more specific term, such as "psychotic illness" and the majority use a larger inclusion of psychiatric disorders. $(7,12)$

For instance, in Ireland, the implementation of Mental Health Act of 2001 in November 2006 improved the legislation referring to mental health, with the purpose of protecting the basic rights of patients involuntarily admitted. For instance, personality disorders in Ireland are no longer diagnoses considered as a criterion of inclusion for involuntary admission in psychiatric institutions, nor the patients who are socially deviant or dependent on alcohol or drugs. (13)

In the UK, a retrospective analysis of coercion measures in England shows that, in 1996, involuntary admissions occupied $23 \%$ of the psychiatric hospital beds, and 10 years later in 2006, involuntary admissions occupied 36\% of the psychiatric hospital beds. Numerous such admissions have been more frequently encountered in patients with a diagnosis of psychotic disorder, organic mental disorders, dementia and substance abuse (14). Substance abuse, according to the literature, was among the main criteria for requesting involuntary admission.

Therefore, in order to better classify and regulate the criteria, as a result of this percentual increase in involuntary admissions, the 2007 Mental Health Act of the UK defines mental disorder as "any disorder or disability of the mind", but the Royal College of Psychiatrists of the UK exclude personality disorders on their own as a criterion for involuntary admission, largely because of the unresponsiveness to available treatments. (7) Also excluded are types of mental illness without psychotic symptoms, noncompliance to treatment, substance abuse and sexual psychological disorders.

In Switzerland, involuntary admission is governed by the Swiss Civil Code, Art ZGB $397 \mathrm{a}$, according to which, patients who may be involuntarily admitted to a psychiatric institution are the following categories: alcoholics, substance addiction, those with learning disabilities and incapacity for selfconduction. Switzerland, compared with other European countries, has the highest rate of involuntary admissions (15), the rate being estimated at about $30 \%$ of the total number of involuntary admissions in Europe for several years.

In Norway, the term "serious mental disorder" is used, and includes active psychosis or other states of mental deficiency where the decrease in functional status is as marked as that seen in psychosis. (7)

In Croatia, the first law on mental health was implemented in 1998. Subsequently, a new law was introduced in 2002 as a result of evaluating various data on the practice of involuntary admissions, performed in conformity with ICD-10 diagnostic criteria. Patients belonging to the diagnosis category of schizophrenia or other psychotic disorders experienced the highest rate of involuntary admissions (4, 16). Although rates of involuntary admissions in the period 19981999 increased significantly (from $30.8 \%$ to $39.6 \%$ ), in 2000-2002, there was a significant decrease in the rate of involuntary admissions of patients with psychiatric disorders, from $5.6 \%$ to $3.5 \%$. (16) 
(1)

In Greece, at the national level, the involuntary admission of people with mental disorders is carried out in accordance with the 2071 Law, enacted to align with European norms. The 2071 law replaced the previous law governing involuntary admission in 1992. $(17,26)$ Prior to the introduction of the new legislation, studies in Greece showed a very high rate of involuntary admissions, so that in 1979 the admission rate was $97 \%$. The current figures for involuntary admissions in Greece show a significant decrease of 40-50\%. (17) In Greece, for admission without consent to the psychiatric hospital, the individual has to meet certain criteria: he or she is suffering from a mental disorder; due to the mental disorder, the patient is not able to care for himself or herself; the acute episode requires treatment, otherwise it may result in self-harm $(18,26)$ or harm to others $(17)$.

In Canada, "mental disorder" is deemed a psychiatric disorder requiring treatment and seriously impacts the capacity of reaction to a person's environment, or to relate with others, and requires constant care and supervision to prevent substantial mental or physical deterioration, or for the protection of the person or others. In the Mental Health Act amendment of 1998, mental deficiency is no longer included in this category. $(7,19)$

In the USA the patient must suffer from a psychiatric illness or disorder that substantially affects emotional functions, cognitive processes and that largely modifies behavior, impairs the patient's capacity of discernment, or the capacity to recognize reality. Involuntary detention can be permitted for individuals with any kind of mental deficiency, epilepsy, alcoholism, or drug addiction. (7)
2. Dangerousness criterion

Preventing the patient from doing harm to himself or others is an important consideration for involuntary hospital admission.

The dangerousness criterion accompanies the presence of mental illness as an additional criterion in many countries for involuntary admission. Sometimes it is sufficient on its own in states such as Finland, Greece, Ireland, Portugal and UK as opposed to the alternative criterion of needing treatment in countries like UK, Denmark, Ireland, Australia, New Zealand, or Hong Kong Special Administrative Region. In Italy, Spain and Sweden, danger to oneself or to others is not considered as a criterion. $(7,20)$

In Canada, some jurisdictions use the dangerousness criterion (Ontario, Nunavut, Northwest Territories, and Quebec), but in others, there is a deterioration criterion. In British Columbia the involuntary admission is offered to "prevent the person's...substantial mental or physical deterioration" or "for the protection of the person...or the protection of others". (7, 19) In Romania, involuntary admission occurs when the psychiatrist considers the criteria for this form of admission to be met. The criteria of nonvoluntary hospitalization according to the Romanian mental health law include selfharm through suicidal thoughts, suicide attempts, food refusal; verbal and / or physical aggression toward others and hallucinatory-delusional symptomatology. (2) Coercive measures in psychiatric care refer to the restriction of the patient's freedom, including measures of confinement and isolation, according to art. 20 of Law $487 / 2002$. Contention measures, according to the mental health law, are needed in situations of self-harm or aggression toward others. A patient with contentious measures may be totally immobilized in both legs or at the level 


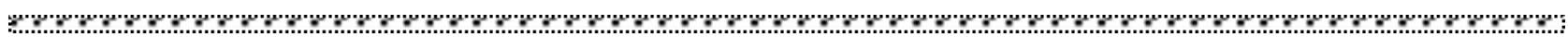

of one leg, either arms or just one arm, but the procedure should not cause bodily injury or pain.

\section{Need for treatment}

In the past, it was generally considered that any psychiatric patient in need of treatment may be subjected to involuntary admission. However, considering the evolution of the structure and content of the many Mental Health Acts and Laws meant to protect the rights of the psychiatric patients, and in the view of respecting the principle of autonomy, a better guideline was needed in order to define the need for treatment criterion on its own and in relation to the other criteria for compulsory admission. The WHO Principles for the Protection of Persons with Mental Illness (or MI Principles, 1991), included in the Resource Book on Mental Health, Human Rights and Legislation, consider the "need for treatment" criterion as applicable "in the case of a person whose mental illness is severe and whose judgment is impaired, failure to admit or retain that person is likely to lead to a serious deterioration in his or her condition or will prevent the giving of appropriate treatment that can only be given by admission to a mental health facility". (12) The approach to this indication varies in all European countries. For instance, Italy best respects this by allowing involuntary admission if a patient who needs urgent treatment that cannot be provided outside a mental health facility. In contrast, in the UK, the patient can only be admitted without consent for treatment in a psychiatric facility if appropriate medication is available. (7)

In North America, some Canadian provinces have the "need for treatment" criterion as required for eligibility to be involuntarily admitted, but others do not, including the two largest provinces, Ontario and Quebec. In the USA there is a strong tendency to replace "need for treatment" with a "dangerousness criterion". $(7,21)$

4. The procedures for involuntary admission in a psychiatric facility

In the cases of involuntary admission, there are various intermediate elements that conduct the protocol for conducting them appropriately. The presenting of the patient resulting in an involuntary hospitalization depends on both the applicants that solicit it and the assessors of eligibility of the patient for the procedure. The legal framework for this varies in different states and is addressed in the respective Mental health Acts. There are a multitude of factors considered, among which are cultural and familial traditions and beliefs, socio-economic status, availability and training of medical personnel, jurisdictions of authorities and enforcement agencies.

Deciding who is the most appropriate person to make an application for the involuntary psychiatric admission of a patient is a difficult matter. It can be a family member, a relative, a guardian in the case of minors, a psychiatrist or a member of the authorities. In Ireland, the request for involuntary admission is done by the spouse or other relative, authorized officer, police member. (13)

On every admission the patient has the right to a mental health court and a legal representative for free legal support. The court-appointed consultant psychiatrist is required to prepare a report within 14 days following a medical examination. The mental health commission consists of an attorney, a consultant psychiatrist and a third party. (13)

In Switzerland, the decision to admit involuntarily a person with mental disorders can be taken by the tutelary association in the region where the individual comes from. However, in the Zurich Canton, for psychiatric assistance, all physicians are 
(1)

authorized to dispose of involuntary admissions of people with mental disorders (15).

There are multiple differences between East Asia and the Western world. In Turkey, for instance, an important emphasis is placed on the primordial role of the patient's family in making decisions. Members of multiple communities, patients with mental disorders participate in community decisions and involve the community in individual decisions. (1)

In Greece, there are two procedures that can lead to hospitalization of the patient with mental disorders. Emergency hospitalization in Greece must be backed by two psychiatric assessments made by approved psychiatrists. Following these assessments, the family members of the patient with a psychiatric disorder submit these two assessments to the authorized service attorney. Subsequently, the authorized prosecutor issues a mandate to the police to escort the patient with a psychiatric disorder to the psychiatric hospital. Another alternative would be for the family to request to hospitalize the patient with a psychiatric disorder directly to the authorized service attorney, without psychiatric assessments. In this case the patient is escorted by the police to the psychiatric hospital on-call. In situations where the subject has no relatives to make the request for involuntary admission, the prosecutor himself may request admission in writing, following a notification by police or a member of the public According to the 2071 Act, when the individual reaches the psychiatric on-call service, the subject is evaluated by two qualified psychiatrists. (17)

In Romania, involuntary admission of an individual with psychiatric disorders may be requested by the attending psychiatrist, family doctor, family member of the individual, police representatives, fire brigades, gendarmes, and public officials, prosecutor or the civil court. The transport of psychiatric patients occurs by ambulance, but in situations of patient aggression, it can be done by firefighters, gendarmes or police, respecting the dignity and integrity of the individual. (26)

\section{DISCUSSIONS}

The practice of involuntary admission of the patient with mental disorders and mental health legislation varies considerably from one country to another in Europe and the rest of the world. Studies on the legal regulations and best practices in psychiatric care, in particular the involuntary admission of the patient with psychiatric disorders, are few. The differences in concept regarding the necessary clinical criteria of a mental disorder to be included on the list of diagnoses subjectable to involuntary admissions create an uneven ethical and moral picture regarding the status of the psychiatric patient amongst the different legislation frameworks, thus predisposing him to other possible issues, such as discrimination.

From the point of view of the legal prognosis, voluntarily admitted patients have a better prognosis than those subjected to involuntary hospitalization, so the latter require greater attention in clinical practice and medical research. An important aspect in the form of involuntary admission of the patient with psychiatric disorders is the maintenance of a balance between the right to selfdetermination and care duties of the medical personnel. Reducing coercive measures such as isolation, contention, non-pharmacological methods is a challenge in psychiatric care.

Homicide and suicide among psychiatric patients in many studies show that mental disorders play a major role in the aggression behaviors of individuals. Some psychiatric disorders related to aggression are depressive disorders, substance abuse, psychosis. 


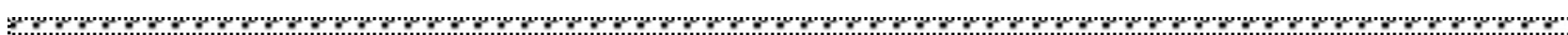

Preventing these events involves identifying and treating these individuals (22). This is achieved by instating the dangerousness criteria as a pillar of psychiatric involuntary admission in many countries.

Some argue that dangerousness criteria should not be instated to counter the loss of discernment arising from mental health legislation, as they unfairly stigmatize, and they spread the notion that any mentally ill person might become violent across the whole population of mentally ill patients.

This insurance measure comes with a cost, as involuntary retention is positively associated with increased severity of the patient's symptomatology, especially aggressive behaviors $(3,23)$. Another aspect to discuss is the fine line between threatened or actual harm inflicted by the patient upon himself or others, which emphasizes the necessity of qualified mental health practitioners to evaluate the patient and instating more than one psychiatrist to confirm the eligibility for compulsory admission.

The long list of entities allowed to direct the patient for involuntary admission predisposes him or her for issues of confidentiality as some of them are present or are required to be present for the psychiatric evaluation. For example, an ethical issue in the Greek health system is the police presence in the psychiatric assessment. Police officers have a duty to fulfill their service obligations, to comply with instructions given by service attorneys, but they are not obliged by law to keep the confidentiality of the person with mental disorders. Cases in which individuals are verbally or physically violent with police officers, psychiatrists or other medical staff, are considered exceptions in which police can participate in psychiatric assessment. $(17,26)$ Police presence in psychiatric assessment in rural areas is motivated by shortage of medical personnel accompanied by fear for the personal safety of psychiatrists (17). By accepting the presence of police officers in psychiatric assessment, besides exceptional situations, the psychiatrist undermines the doctor-patient relationship and the importance of confidentiality of patient data.

An important aspect that increases the voluntary addressability of mental health services is the involvement of patients with mental disorders in the management of the disease, especially when they have no acute affections and have more knowledge about their disorder. A possible solution to consider the patient's options would be the Psychiatric Advance Directive, implemented already in some countries.

The Advance Directive is an act whereby individuals can manifest certain desires that need to be respected when they no longer have the mental capacity to decide for themselves. Since 2008, in the Netherlands (24), the advance directive has been included in mental health acts. This directive includes the patient's desire to be hospitalized against his will in situations of decompensation of the disease for protective and therapeutic purposes (25). It is a statement in which the individual can express certain treatment preferences to be applicable when his capacity for decision is diminished or absent (27).

\section{CONCLUSIONS}

From a comparison analysis of various involuntary admission procedures, a need to improve psychiatric care and protocol is arising. Some points of improvement would include providing the patients with mental disorders with as much information as possible related to the reasons and duration of hospitalization and protecting patients' rights during hospitalization 

Coercive measures in psychiatric care through the involuntary form of admission are ethically and legally justified in psychiatric emergencies when there is danger of self-harm and aggression toward others.

The value of confidentiality in the therapeutic relationship with the psychiatric patient is recognized by many medical staff as being fundamental in psychiatric practice. Breaching the confidentiality of the medical act involving the psychiatric patient predisposes to social consequences of marginalization, stigmatization, discrimination which in turn causes important decreases of the patient's self-esteem, even the impossibility of social reinsertion and impairment of the level of social, personal and professional functioning. The presence of police members at psychiatric assessments prior to admission is considered to be an unethical aspect of psychiatric care due to police officers not being required to preserve the confidentiality of patients with psychiatric disorders. Maintaining the confidentiality and reducing the effects of stigmatization and discrimination are goals towards which a multitude of entities have to dedicate their work, such as services of communitarian psychiatry, mental health centers outside of the hospital setting, social workers, government institutions tasked with regulation of employment, authorities.

In accordance to the European tendencies and norms regarding involuntary admission in psychiatric patients, Romanian Mental health law updated its 487/2002 mental health act through law $129 / 2012$, bringing new modifications with the intention of attaining the professional standards imposed by WHO and aligning itself with the improvements implemented across the other European countries.

Professionals involved in the therapy of psychiatric patients believe that the premises of therapeutic success are met by a strong ethical foundation, such as upholding the four ethical principles of autonomy, nonmaleficence, beneficence and justice.

\section{ACKNOWLEDGEMENTS AND DISCLOSURES}

The authors declare that they have no potential conflicts of interest to disclose.

\section{REFERENCES}

1. Fistein, E. C., Holland, A. J., Clare, I. C. H., \& Gunn, M. J. (2009). A comparison of mental health legislation from diverse Commonwealth jurisdictions. International Journal of Law and Psychiatry, 32(3), 147-155.

2. Crăciun P, Astărăstoae V, Turliuc Ş, Vicol M-C. Non-voluntary admission of patients with mental disorders: ethical and social issues. Revista de cercetare şi intervenţie socială 2013; 42: 310-319.

3. Crăciun P, Crăciun G, Chiriţă R, Radu A. Ethical and legal issues in drug addiction. Bulletin of Integrative psychiatry $2011 ; 48(1): 24-28$.

4. Crăciun P, Vicol MC, Astărăstoae V. The Involuntary admission of psychiatric patient: biopsychosocial issues. Logos, Universality, Mentality, Education, Novelty, Section: Philosophy and Humanistic Sciences 2013; 1: $179-187$.

5. Crăciun P, Vicol MC, Turliuc Ş, Astărăstoae V. Autonomy versus paternalism in non-voluntary admission. Romanian Journal of Bioethics 2012; 10(4): 92-101.

6. Crăciun P, Crăciun G, Nechita DC. The phenomenon of recreaţional use of psychoactive substances (a brief update). Buletin de Psihiatrie Integrativă 2012; 54(3): 38-43.

7. Zhang S, Mellsop G, Brink J, Wang X. Involuntary admission and treatment of patients with mental disorder, Neurosci Bull, January 2015.

8. Swanson JW, Swartz MS, Elbogen EB et al. Effects of involuntary outpatient commitment on subjective quality of life in persons with severe mental illness. Behav Sci Law 2003; 21(4): 473-491. 
о

9. Wright ER, Gronfein WP, Owens TJ. Deinstitutionalization, social rejection, and the self-esteem of former mental patients. J Health Soc Behav 2000; 41(1): 68-90.

10. Link BG, Struening EL, Neese-Todd $\mathrm{S}$ et al. Stigma as a barrier to recovery: the consequences of stigma for the self-esteem of people with mental illness. Psychiatr Serv 2001; 52(12): 1621-1626.

11. World Health Organization. Mental Health Care Law: Ten Basic Principles. Geneva: World Health Organization, 1996.

12. Freeman M, Pathare S. WHO Resource Book on Mental Health, Human Rights and Legislation. Geneva: World Health Organization, 2005.

13. Latif Z, Malik MA. Mental health legislation in Ireland: a lot done, more to do. J Am Acad Psychiatry Law 2012; 40(2): 266-269.

14. Molodynski A, Rugkåsa J, Burns T. Coercion and compulsion in community mental health care. Br Med Bull 2010; 95: 105-119.

15. Lay B, Nordt C, Rössler W. Variation in use of coercive measures in psychiatric hospitals. Eur Psychiatry 2011; 26(4): 244-251.

16. Kozumplik O, Jukic V, Goreta M. Involuntary hospitalizations of patients with mental disorders in Vrapce psychiatric hospital. Five years of implementation of the first Croatian law on protection of persons with mental disorders. Croat Med J 2003; 44(5): 601-605.

17. Douzenis A, Michopoulos I, Economou M et al. Involuntary admission in Greece: A prospective national study of police involvement and client characteristics affecting emergency assessment. Int J Soc Psychiatry 2012; 58(2): 172177.

18. Carter GL, Safranko I, Lewin TJ et al. Psychiatric hospitalization after deliberate self-poisoning. Suicide Life Threat Behav 2006; 36(2): 213-222.

19. Rodrigues R, MacDougall AG, Zou G, Lebenbaum M, Kurdyak P, Li L, Shariff SZ, Anderson K. Involuntary hospitalization among young people with early psychosis: A population-based study using health administrative data. SCHRES-08215;

20. Sal ize HJ, Dressing H. Epidemiology of involuntary placement of mentally ill people across the European Union. Br J Psychiatry 2004, 184: 163-168.

21. Gray J E, McSherry BM, O’Reilly RL, Weller PJ. Australian and Canadian mental health Acts compared. Aust N Z J Psychiatry 2010, 44: 1126-1131.

22. Roma P, Pazzelli F, Pompili M et al. Mental Illness in homicide-suicide: a review. J Am Acad Psychiatry Law 2012; 40(4): 462-468.

23. Kaltiala-Heino R. Involuntary commitment and detainment in adolescent psychiatric inpatient care. Soc Psychiatry Psychiatr Epidemiol 2010; 45(8):785-793.

24. van der Post L, Mulder CL, Bernardt CML et al.Involuntary admission of emergency psychiatric patients: report from the Amsterdam Study of Acute Psychiatry. Psychiatr Serv 2009; 60(11): 1543-1546.

25. Berghmans R, van der Zanden M. Choosing to limit choice: Self-binding directives in Dutch mental health care. Int J Law Psychiatry 2012; 35(1): 11-18.

26. Bilanakis N. Psychiatric Care and Human Rights in Greece. Athens: Odysseas, 2004

27. Papageorgiou A,$\underline{\text { King M }}$, Janmohamed A et al. Advance directives for patients compulsorily admitted to hospital with serious mental illness: Randomised controlled trial. Br J Psychiatry 2002; 181: 513-519.

\section{Correspondence:}

Mirona Letiţia Dobri-Resident in Psychiatry, +40724554270, mirona.dobri@gmail.com

Submission: 28 may 2019

Acceptance: 15 aug 2019 